\title{
Comparación de pacientes cocainómanos y heroinómanos en el EuropASI
}

\author{
Olaya García Rodríguez; Roberto Secades Villa; José Ramón Fernández Hermida; \\ José luis Carballo Crespo; José María Errasti Pérez; Susana Al-Halabi Díaz
}

Grupo de Conductas Adictivas. Departamento de Psicología. Universidad de Oviedo.

\section{RESUMEN}

El objetivo de este estudio es analizar las diferencias entre heroinómanos y cocainómanos en las áreas que mide el EuropASI. Se pretende, a su vez, elaborar un perfil de usuario para cada uno de los grupos con las características psicosociales de estos pacientes. La muestra se compuso de 373 pacientes extraídos de forma aleatoria de entre todas las personas que solicitaron tratamiento por su adicción a la cocaína o a la heroína durante el año 2003 en los centros de Proyecto Hombre en España. Los resultados muestran que, en general, los usuarios del programa de heroína presentan mayor gravedad en su patrón de consumo y padecen más problemas asociados que los usuarios del programa de cocaína. Esta tendencia se ve reflejada prácticamente en todas las áreas que mide el EuropASI: estado de salud, educación y empleo, situación legal y relaciones sociales/familiares. El estado psiquiátrico es el único área en el que no se encuentran diferencias entre ambos grupos. Se discuten los resultados y se analizan las implicaciones clínicas de los mismos.

Palabras clave: EuropASI, cocainómanos, heroinómanos, perfil psicosocial.

\begin{abstract}
The objective of this study is to analyse the differences between heroin addicts and cocaine addicts in the areas measured by the EuropASI. It is also aimed to draw up a user profile for each of the groups, with the psychosocial characteristics of these patients. The sample was made up of 373 patients, taken at random from those requesting treatment for addiction to cocaine or heroin during 2003 at the Proyecto Hombre centres in Spain. The results show that, in general, the heroin programme users present greater severity in their consumption pattern and have more associated problems than the cocaine programme users. This tendency is reflected in practically all the areas measured by the EuropASI: state of health, education and employment, legal situation and social/ family relationships. Psychiatric state is the only area in which there are no differences between the two groups. The results are discussed and their clinical implications analysed.
\end{abstract}

Key words: EuropASI, cocaine addicts, heroin addicts, psychosocial profile.

\section{INTRODUCCIÓN}

E EuropASI (Kokkevi y Hartgers, 1995) es la adaptación europea del Addiction Severity Index (ASI) (McLellan, Luborsky, Woody y O’Brien, 1980), uno de los instrumentos más utilizados en la práctica clínica diaria para evaluar el consumo de drogas y los problemas asociados en personas con problemas de adicciones. Se trata de una entrevista semiestructurada y estandarizada que proporciona información sobre aspectos de la vida del paciente con problemas de abuso o dependencia de sustancias que han podido contribuir al desarrollo de su adicción.

Las propiedades psicométricas del ASI han quedado probadas a través de multitud de estudios
(Brown, Seraganian y Shields, 1999; Chermack et al., 2000; Leonhard, Mulvey, Gastfriend y Shwartz, 2000; Scheurich et al., 2000) por lo que se puede afirmar que se trata de un instrumento válido y fiable. Existen además versiones autoaplicadas (Rosen, Ouimette, Sheikh, Gregg y Moos, 2002) e informatizadas (Butler et al., 2001) de este instrumento que han demostrado también su consistencia y aplicabilidad.

Desde el punto de vista clínico, se ha utilizado para describir las necesidades de los pacientes al inicio del tratamiento, asignar a los pacientes a las estrategias terapéuticas adecuadas y evaluar los resultados de las intervenciones (French, Salome, Sindelar y McLellan, 2002). En relación con esto, el ASI es un buen instrumento para valorar la eficacia de los programas de tratamiento y determinar los resultados de éstos en 
función de la mejoría de los pacientes y, también, en función de los costes-beneficios (Ballard, 2003; Johnson, Wiechelt, Ahmed y Schwartz, 2003; Sindelar, Jofre-Bonet, French y McLellan, 2004).

Por otra parte, existen algunos estudios que han utilizado los resultados de pacientes en el ASI para tratar de averiguar qué áreas de este instrumento son las que mejor predicen el éxito en el tratamiento. En el estudio de Hanlon, O'Grady y Bateman, (2000) se vio cómo las áreas que mejor predecían los resultados en el tratamiento eran la de empleo/soportes y la de uso de drogas. Otros estudios han utilizado los resultados del ASI como variable predictora de retención en el tratamiento (Claus, Kindleberger y Dugan, 2002; McCaul, Svikis y Moore, 2001) y como variable predictora para que personas con problemas de abuso de sustancias soliciten ayuda formal (Siegal, Falck, Wang y Carlson, 2002).

A pesar de la proliferación de trabajos que han empleado el ASI, son escasos los estudios en donde se comparen diferentes tipos de adictos en función de las respuestas a este instrumento. Sin embargo, cocainómanos y heroinómanos han sido comparados tradicionalmente en diferentes tipos de variables. Algunos de estos estudios tratan de probar o refutar la Teoría de la Automedicación comparando ambos grupos en función de variables como la personalidad, el autoconcepto, la depresión, la alexitimia, etc. que puedan predecir la elección de la sustancia (Aharonovich, Nguyen y Nunes, 2001; Craig y Olson, 1990; Fieldman, Woolfolk y Allen, 1995; Shorin, 1999). Otros estudios han prestado más atención a aspectos más objetivos, como las diferencias entre ambos grupos en cuanto a tasas de recaídas (Hubbard, Flynn, Craddock y Fletcher, 2001).

El objetivo de este estudio es analizar las diferencias entre dos muestras significativas de pacientes heroinómanos y cocainómanos en las áreas que mide el EuropASI. Se pretende, a su vez, elaborar un perfil de usuario para cada uno de los grupos que pueda dar una visión general de las características psicosociales más relevantes de estos pacientes.

\section{MÉTODOS}

\section{Muestra}

La muestra se extrajo de forma aleatoria de entre todas las personas que habían solicitado tratamiento por su adicción a la cocaína o a la heroína durante el año 2003 en diferentes centros de Proyecto Hombre en España: Galicia, Baleares, Málaga, Sevilla, Madrid,
Asturias, Murcia, Alicante, Castellón, Burgos, Granada, Huelva, León, Navarra, Valladolid, Guadalajara, Cataluña, La Rioja, Cantabria y Córdoba. Los participantes en el estudio fueron 373 usuarios de los Programas terapéuticos de Proyecto Hombre. De éstos, 213 pacientes habían solicitado tratamiento para sus problemas con la heroína y 160 pacientes lo habían hecho para sus problemas con la cocaína.

La edad media de la muestra total es de 31,85 años (33,2 los usuarios del programa de heroína y 30 los pacientes del programa de cocaína). El rango de edad de estos pacientes estaba entre 20 y 53 años. Respecto al sexo, el $88 \%$ eran hombres y el $12 \%$ mujeres. El 63\% de los pacientes estaban solteros, el $21 \%$ casados y el resto (16\%) separados, divorciados o viudos. El 79,6\% de los pacientes había finalizado la educación básica. La media de años de educación básica cursada fue de 8,5 años. El 18,5\% de pacientes había cursado algún año de educación superior. La media de años de estudios superiores fue de 3,3. El $69,6 \%$ de los pacientes había trabajado a tiempo completo en los últimos 3 años. El 30.4\% había trabajado esporádicamente.

\section{Instrumento}

El EuropASI es una entrevista semiestructurada y heteroaplicada que se utiliza para valorar la gravedad de la adicción de los sujetos con problemas de sustancias. En España se cuenta con la traducción de este instrumento desde hace ya varios años (Bobes, González, Sáiz y Bousoño, 1996).

Consta de 6 áreas que son independientes entre sí: situación médica (16 ítems), situación laboral/ soportes (26 ítems), consumo de alcohol/otras drogas (28 ítems), problemas legales (23 ítems), relaciones familiares/sociales (26 ítems) y estado psiquiátrico (22 ítems). Las áreas problema tienen todas la misma estructura, empezando por una serie de ítems objetivos que proporcionan un cuadro de la situación actual del paciente, y terminando con dos preguntas subjetivas sobre la importancia de los problemas referidos en la entrevista objetiva y del tratamiento para los mismos.

\section{Procedimiento}

Las entrevistas fueron aplicadas durante la fase inicial del programa de tratamiento por personal entrenado para tal fin en los centros de Proyecto Hombre antes mencionados. El tiempo de aplicación de la prueba osciló entre 45 y 60 minutos en función del paciente y de la experiencia del entrevistador. La reco- 
gida de datos se hizo durante el año 2003. De todas las entrevistas recogidas durante ese año se extrajo una muestra representativa de usuarios del Programa de heroína y de usuarios del Programa de cocaína.

\section{Análisis de datos}

Se llevaron a cabo diferentes análisis de frecuencias para describir las características de la muestra total y para obtener el perfil de usuario de cada una de las sustancias. Se realizaron análisis bivariados (chi cuadrado con la corrección de continuidad de Yates para las variables dicotómicas y $t$ de Student para las variables continuas) para comparar ambas muestras y analizar las posibles diferencias entre ellas. Algunas de las variables que son medidas de forma continua en el EuropASI se dicotomizaron para realizar análisis más adecuados. El nivel de confianza fue del 95\% y el paquete estadístico utilizado el SPSS-11.

\section{RESULTADOS}

\section{Perfil de los usuarios de los Programas de cocaína y heroína}

En la Tabla 1 se muestra el perfil psicosocial de los usuarios de ambos programas. Como se puede observar, el perfil del paciente del programa de heroína es más grave tanto en consumo de sustancias como en problemas asociados.

\section{Tabla 1. Perfil psicosocial de los participantes en el estudio}

\begin{tabular}{|c|c|c|}
\hline & Heroína ( $\mathrm{N}=213$ ) & Cocaína (N=160) \\
\hline $\begin{array}{l}\text { Sexo: } \\
\quad \text { Hombres } \\
\text { Mujeres }\end{array}$ & $\begin{array}{l}185(86,9 \%) \\
28(13,1 \%)\end{array}$ & $\begin{array}{c}143(89,4 \%) \\
17(10,6 \%)\end{array}$ \\
\hline Edad media & 33 & 30 \\
\hline $\begin{array}{c}\text { Estado civil: } \\
\text { Casado/a } \\
\text { Divorciado/a } \\
\text { Separado/a } \\
\text { Soltero/a } \\
\text { Viudo/a }\end{array}$ & $\begin{array}{c}33(15,5 \%) \\
7(3,3 \%) \\
27(12,7 \%) \\
145(68,1 \%) \\
1(0,5 \%)\end{array}$ & $\begin{array}{c}48(30 \%) \\
6(3,8 \%) \\
14(8,7 \%) \\
91(56,9 \%) \\
1(0,6 \%)\end{array}$ \\
\hline $\begin{array}{l}\text { Lugar de residencia: } \\
\text { Ciudad Grande }(>100.000) \\
\text { Ciudad Mediana }(10.000-100.000) \\
\text { Ciudad Pequeña }(<10.000)\end{array}$ & $\begin{array}{l}118(55,4 \%) \\
54(25,4 \%) \\
39(18,3 \%)\end{array}$ & $\begin{array}{l}65(40,6 \%) \\
56(35 \%) \\
39(24,4 \%)\end{array}$ \\
\hline Edad de inicio de consumo de la sustancia principal & 19 & 21 \\
\hline $\begin{array}{l}\text { Vía de consumo sustancia principal: } \\
\text { Oral } \\
\text { Nasal } \\
\text { Fumada } \\
\text { Inyectada }\end{array}$ & $\begin{array}{c}1(0,5 \%) \\
10(4,7 \%) \\
117(54,9 \%) \\
86(39,9 \%)\end{array}$ & $\begin{array}{l}5(3,1 \%) \\
120(75 \%) \\
24(15 \%) \\
9(5,6 \%)\end{array}$ \\
\hline $\begin{array}{l}\text { Problemas médicos crónicos: } \\
\text { Sí } \\
\text { No }\end{array}$ & $\begin{array}{l}108(50,7 \%) \\
105(49,3 \%)\end{array}$ & $\begin{array}{c}38(23,8 \%) \\
122(76,2 \%)\end{array}$ \\
\hline $\begin{array}{l}\text { Fuente de ingresos: } \\
\text { Empleo } \\
\text { Familiares, amigos, etc. } \\
\text { Otros(ayuda social, paro, pensión, etc.) }\end{array}$ & $\begin{array}{l}55(24,4 \%) \\
69(32,4 \%) \\
92(43,2 \%)\end{array}$ & $\begin{array}{c}113(70,6 \%) \\
15(9,4 \%) \\
32(20 \%)\end{array}$ \\
\hline Pacientes que han estado en prisión & $83(39 \%)$ & $13(8 \%)$ \\
\hline $\begin{array}{l}\text { Problemas psiquiátricos: } \\
\text { Depresión } \\
\text { Ansiedad } \\
\text { Intentos de suicidio }\end{array}$ & $\begin{array}{l}109(51,2 \%) \\
127(59,6 \%) \\
66(31 \%)\end{array}$ & $\begin{array}{l}75(46,9 \%) \\
98(61,3 \%) \\
35(21,9 \%)\end{array}$ \\
\hline
\end{tabular}




\section{Diferencias entre cocainómanos y heroinómanos}

\section{Uso de alcohol y otras drogas}

Existen diferencias significativas $(p<.05)$ en los años que los pacientes llevan consumiendo las sustancias que mide el EuropASI (alcohol, heroína, metadona/ LAAM, otros opiáceos/analgésicos, benzodiacepinas/ barbitúricos/ sedantes/ hipnóticos, cocaína, anfetaminas, cannabis, alucinógenos e inhalantes). En todos los casos, los heroinómanos han consumido durante más tiempo que los cocainómanos. En cambio, no existen diferencias en cuanto a la edad de inicio de consumo, solamente existen diferencias significativas $(p<.05)$ en la edad de inicio de consumo de anfetaminas, donde los heroinómanos empiezan a consumir antes que los cocainómanos aunque con una diferencia de medias muy pequeña $(18,11$ frente a 18,37). Otra diferencia entre ambas muestras es la vía de administración de cocaína. Los pacientes que acuden a tratamiento por sus problemas con la cocaína refieren un uso principalmente nasal (75\%) mientras que los pacientes cuyo principal problema es la adicción a la heroína refieren un uso fumado o inyectado de cocaína (73\%). Además, un porcentaje mayor de heroinómanos que de cocainómanos $161 \%$ frente a 9\%) refiere haberse inyectado alguna vez algún tipo de sustancia. En el mismo sentido, los usuarios del programa de heroína han sufrido más sobredosis y han acudido a mayor número de tratamientos previos tanto por problemas de alcohol como de otras drogas $(p<.05)$ y han pasado más meses de abstinencia continuada $(12,28$ frente a 3,92) que los de cocaína.
Por último, y en sentido contrario, también existen diferencias significativas $(p<.05)$ en la cantidad de dinero que estas personas gastan en alcohol. En este caso los cocainómanos gastan casi 200 más que los heroinómanos al mes. En cambio, no existen diferencias significativas en la cantidad de dinero gastado en drogas al mes entre ambas muestras.

\section{Situación médica}

Como se observa en la Tabla 2 existen diferencias significativas $(p<.05)$ entre ambas muestras en todos los ítems valorados. Solamente en el número de veces hospitalizados no se encuentran diferencias entre ambos grupos. Tener un problema médico cróni$\mathrm{co}$, haber estado o estar infectado de hepatitis y estar infectado de VIH son los ítems en donde más diferencias encontramos entre cocainómanos y heroinómanos. Tanto en estos ítems como en el resto en los que se encuentran diferencias, son los heroinómanos los que tienen una situación médica más problemática.

\section{Empleo/soportes}

En la Tabla 3 se pueden ver las diferencias entre ambas muestras en la educación recibida, empleo y desempleo, fuente de ingresos y personas a cargo del paciente. Mientras que no se encuentran diferencias significativas en el número de años de educación básica, sí existen diferencias en cuanto al número de años de educación superior. En este caso, los cocainómanos habían recibido más años de educación superior que los heroinómanos. Tampoco hay diferencias en el periodo de tiempo más largo de empleo

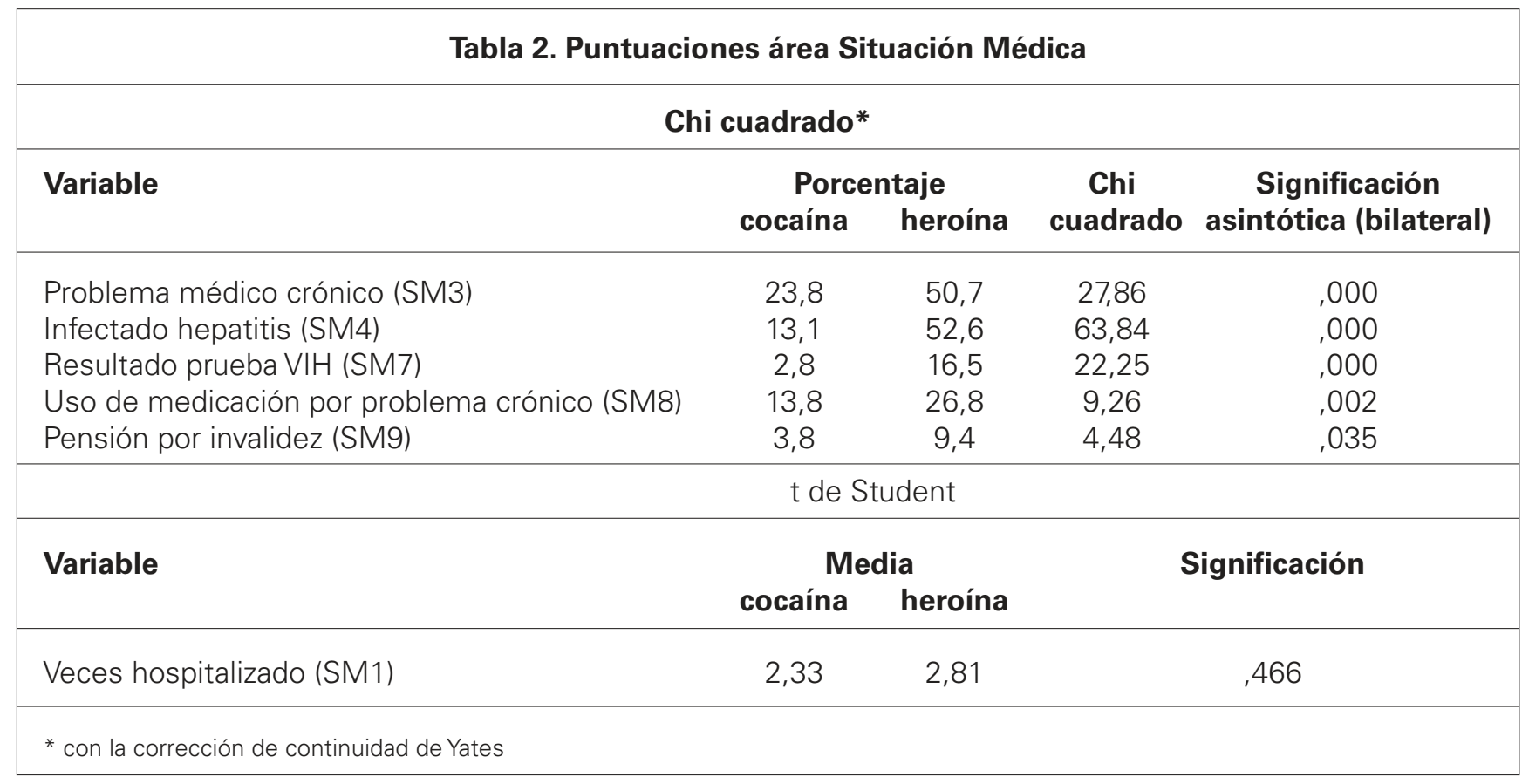


Tabla 3. Puntuaciones área Empleo/soportes

\begin{tabular}{|c|c|c|c|c|}
\hline \multicolumn{5}{|c|}{ t de Student } \\
\hline \multirow{2}{*}{\multicolumn{2}{|c|}{ Variable }} & \multicolumn{2}{|c|}{ Media } & \multirow[t]{2}{*}{ Significación } \\
\hline & & cocaína & heroína & \\
\hline Años educación básica (ES1) & & 9,04 & 8,17 & 240 \\
\hline Años educación superior (ES2) & &, 76 &, 43 &, 000 \\
\hline Periodo más largo de empleo (en meses) ( & S5) & 70,90 & 47,76 & 052 \\
\hline Periodo más largo de desempleo (en mese & ) (ES6) & 9,75 & 24,22 &, 000 \\
\hline Personas que dependen de él/ella (ES 20) & &, 67 &, 54 & 257 \\
\hline \multicolumn{5}{|c|}{ Chi cuadrado* } \\
\hline Variable & $\begin{array}{r}\mathrm{Po} \\
\text { cocaín }\end{array}$ & $\begin{array}{l}\text { entaje } \\
\text { heroína }\end{array}$ & $\begin{array}{l}\text { Chi } \\
\text { cuadrado }\end{array}$ & $\begin{array}{l}\text { Significación } \\
\text { asintótica (bilateral) }\end{array}$ \\
\hline Empleo como fuente de ingresos (ES18) & 74,4 & 28,5 & 75,44 &, 000 \\
\hline
\end{tabular}

regular pero sí en el periodo más largo de desempleo. Son los heroinómanos los que más tiempo han pasado desempleados. Por último, también se encuentran diferencias entre ambos grupos en la principal fuente de ingresos que tienen los pacientes. Los cocainómanos suelen referir ingresos por empleos mientras que los heroinómanos refieren más otro tipo de fuentes de ingresos (compañeros, familiares o amigos, paro, ayuda social, pensión o seguridad social...).

\section{Situación legal}

Como se puede ver en la Tabla 4, existen diferencias significativas $(p<.05)$ entre cocainómanos y heroinómanos en casi todos los ítems del área de situación legal. Los únicos delitos en los que no se encuentran diferencias significativas son: prostitución, conducir embriagado y delitos de tráfico (velocidad, conducir sin carné, conducción peligrosa, etc.). En el resto de delitos que valora el EuropASI (posesión y tráfico de drogas, delitos contra la propiedad, delitos violentos y alteración del orden o vagabundeo) se encuentran diferencias entre ambas muestras. En todos los casos, un mayor número de heroinómanos que de cocainómanos habían sido acusados de cometer estos delitos.

\section{Relaciones familiares/sociales}

En la Tabla 5 se pueden ver las diferencias entre ambas muestras en el área de relaciones familiares y sociales. Los heroinómanos tienden más a compartir el tiempo libre con personas con problemas de dro- gas que los cocainómanos $(\mathrm{p}<.05)$. A pesar de esto, no existen diferencias en cuanto a la satisfacción del uso del tiempo libre entre las dos muestras. Tampoco se encuentran diferencias en haber mantenido relaciones próximas, duraderas y personales con los padres, hermanos, parejas, hijos o amigos de los pacientes de ambos grupos, pero sí en cuanto a las relaciones con las madres. Un mayor número de heroinómanos que de cocainómanos refieren haber tenido una relación próxima con su madre. Por último, sí existen diferencias significativas en haber tenido problemas con madres, padres, hermanos, parejas, otros familiares y vecinos. En todos estos casos, son los heroinómanos los que más refieren este tipo de problemas. En cambio, no existen diferencias entre cocainómanos y heroinómanos en cuanto a los problemas con los hijos, amigos y compañeros de trabajo.

\section{Estado psiquiátrico}

Como se puede ver en la Tabla 6, solamente se encuentran diferencias significativas $(p<.05)$ en que un mayor número de heroinómanos que de cocainómanos (31\% frente a $22 \%$ ) ha intentado suicidarse. En el resto de variables que mide el EuropASI no existen diferencias significativas entre ambos grupos. Estas variables son las siguientes: cobro de pensión por problemas psiquiátricos, uso de medicación por problemas psiquiátricos, haber sufrido diferentes trastornos psicopatológicos sin que estos estuviesen directamente relacionados con el uso de alcohol o drogas y, por último, haber experimentado ideación suicida. 


\begin{tabular}{|c|c|c|c|c|}
\hline \multicolumn{5}{|c|}{ Chi cuadrado* } \\
\hline Variable & $\begin{array}{l}\text { Porc } \\
\text { cocaína }\end{array}$ & $\begin{array}{l}\text { taje } \\
\text { heroína }\end{array}$ & $\begin{array}{c}\text { Chi } \\
\text { cuadrado }\end{array}$ & $\begin{array}{l}\text { Significación } \\
\text { asintótica (bilateral) }\end{array}$ \\
\hline Ingreso motivado por causas judiciales (SL1) & 1,9 & 11,7 & 12,79 & .000 \\
\hline Libertad condicional (SL2) & 5 & 10,8 & 4,03 & 045 \\
\hline Acusado de posesión y tráfico de drogas (SL3) & 17,5 & 38,5 & 19,37 &, 000 \\
\hline Acusado de delitos contra la propiedad (SL4) & 13,8 & 58,2 & 75,83 &, 000 \\
\hline Acusado de delitos violentos (SL5) & 11,3 & 20,7 & 5,83 & ,016 \\
\hline Acusado de otros delitos (SL6) & 3,8 & 12,7 & 9,02 &, 003 \\
\hline Condenado por algún delito (SL7) & 12,5 & 50,2 & 57,93 & ,000 \\
\hline Acusado de alteración orden, vagabundeo (SL8) & 6,9 & 20,7 & 17,80 &, 000 \\
\hline Acusado de prostitución (SL9) & 0 & 0,5 & 0,75 & ,386 \\
\hline Acusado de conducir embriagado (SL10) & 16,3 & 11,7 & 1,57 & 210 \\
\hline Acusado de delitos tráfico (SL11) & 22,5 & 23 & 0,01 & 909 \\
\hline Pendiente cargos (SL 15) & 18,8 & 34,3 & 11,01 & , 001 \\
\hline \multicolumn{5}{|c|}{ t de Student } \\
\hline \multirow[t]{2}{*}{ Variable } & \multicolumn{2}{|c|}{ Media } & \multirow{2}{*}{\multicolumn{2}{|c|}{ Significación }} \\
\hline & cocaína & heroína & & \\
\hline Meses en la cárcel (SL12) & 92 & 16,12 & \multirow{2}{*}{\multicolumn{2}{|c|}{$\begin{array}{l}, 000 \\
.000\end{array}$}} \\
\hline Último periodo en la cárcel (SL13) & 80 & 7,49 & & \\
\hline
\end{tabular}

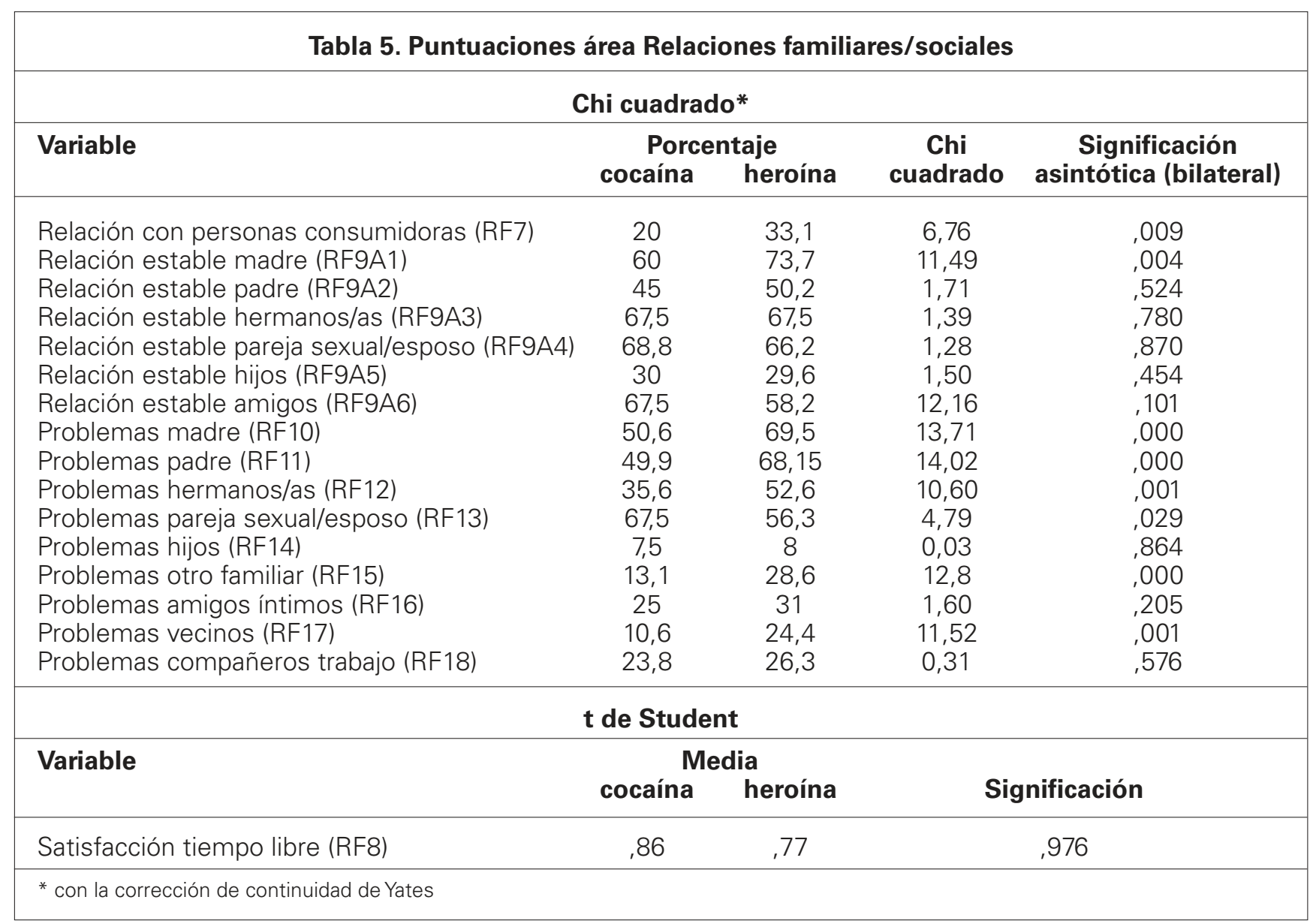


Tabla 6. Puntuaciones área Estado psiquiátrico

\begin{tabular}{|c|c|c|c|c|}
\hline \multicolumn{5}{|c|}{ Chi cuadrado* } \\
\hline Variable & $\begin{array}{l}\text { Porce } \\
\text { cocaína }\end{array}$ & $\begin{array}{l}\text { ntaje } \\
\text { heroína }\end{array}$ & $\begin{array}{c}\text { Chi } \\
\text { cuadrado }\end{array}$ & $\begin{array}{l}\text { Significación } \\
\text { asintótica (bilateral) }\end{array}$ \\
\hline Pensión psiquiátrica (EP2) & 1,9 & 5,2 & 2,73 & 099 \\
\hline Depresión severa (EP3) & 46,9 & 51,2 & 0,67 & ,412 \\
\hline Ansiedad o tensión severa (EP4) & 61,3 & 59,6 & 0,10 & 751 \\
\hline Problemas para comprender/recordar (EP5) & 42,5 & 46,0 & 0,45 &, 500 \\
\hline Alucinaciones (EP6) & 20,6 & 18,3 & 0,31 &, 576 \\
\hline Problemas para controlar conductas violentas (EP7) & 39,4 & 46,0 & 1,63 & 201 \\
\hline Medicación por problemas psiquiátricos (EP8) & 30,6 & 36,2 & 1,24 & 265 \\
\hline Ideación suicida (EP9) & 39,4 & 44,6 & 1,02 &, 313 \\
\hline Intentos de suicidio (EP10) & 21,9 & 31,0 & 3,84 &, 050 \\
\hline \multicolumn{5}{|c|}{ t de Student } \\
\hline \multirow[t]{2}{*}{ Variable } & \multicolumn{3}{|c|}{ Media } & \\
\hline & & caína & heroína & Significación \\
\hline Tratamiento hospitalario problema psiquiátrico (EP1) & & 1,11 & 190,57 &, 122 \\
\hline Tratamiento ambulatorio problema psiquiátrico (EP2) & & 3,36 & 180,42 & 168 \\
\hline
\end{tabular}

\section{DISCUSIÓNY CONCLUSIONES}

El objetivo de este estudio era analizar las diferencias entre dos muestras de pacientes heroinómanos y cocainómanos en cada una de las áreas que mide el EuropASI. A su vez, se pretendía elaborar un perfil psicosocial de los pacientes que acuden a tratamiento por sus problemas con la heroína y de los que acuden a tratamiento por sus problemas con la cocaína en los centros de Proyecto Hombre de España.

El perfil de usuario del programa de heroína es un varón de unos 33 años, soltero, que vive en una gran ciudad en residencia propia o de su familia. Comenzó a consumir heroína a los 19 años y medio y generalmente la consume fumada. Se le puede considerar un policonsumidor ya que, además de heroína, suele consumir alcohol en grandes dosis, cocaína, cannabis, benzodiacepinas (BZD), barbitúricos, sedantes o hipnóticos. Lleva unos 12 años consumiendo heroína y unos 10 años con el patrón de policonsumo. Ha tomado metadona en alguna ocasión y, si ha acudido a tratamiento, ha sido a un programa sustitutivo ambulatorio. Padece algún problema médico crónico que le ha hecho estar hospitalizado en varias ocasiones y alguna vez ha experimentado ansiedad, depresión o problemas para controlar su conducta violenta. Tiene completados los estudios básicos y trabaja a tiempo completo, pero su fuente de ingresos principal proviene de compañeros, familiares o amigos. Gasta grandes cantidades de dinero en drogas. Ha tenido algún problema legal llegando a ser condenado en alguna ocasión.

El perfil de usuario del programa de cocaína de Proyecto Hombre es un varón de unos 30 años, soltero, que vive en una gran ciudad y en residencia propia. Empezó a consumir cocaína a los 21 años por vía nasal y aparte de esta sustancia consume habitualmente alcohol y cannabis. Suele gastar grandes cantidades de dinero en este consumo y, por lo general, no tiene problemas legales importantes motivados por el consumo de drogas. Acude a tratamiento por primera vez tras unos 7 años de consumo y no tiene problemas médicos relevantes, aunque en ocasiones ha experimentado ansiedad y depresión. Tiene completados los estudios básicos y su fuente de ingresos es un puesto de trabajo a tiempo completo.

Los pacientes que acuden a tratamiento por su problema con la heroína suelen presentar un patrón de policonsumo más marcado que aquellos que acuden a tratamiento por cocaína. En la misma línea, la media de edad de las personas que acuden a tratamiento a Proyecto Hombre es 3 años superior en los usuarios del programa de heroína que en el de cocaína. Por otra parte, el paciente que acude a tratamien- 
to por heroína suele tener un historial más extenso de tratamientos anteriores que el que acude por problemas con la cocaína.

Los pacientes del tratamiento tradicional presentan mayores problemas en el estado de salud que los del Programa de cocaína. En general, tienen más problemas médicos crónicos que necesiten de medicación prescrita y, a su vez, hay un mayor número de pacientes que están infectados de VIH y/o de hepatitis. Estos datos coinciden con los encontrados en otras investigaciones en las que se valoraba el estado médico y legal de adictos a sustancias (Serrat More, 2003).

En el área de empleo y soportes no existen diferencias significativas en cuanto a educación básica, pero sí en cuanto a educación superior. Hay más personas que han cursado algún año de educación superior entre las que acuden a tratamiento por sus problemas con la cocaína que entre las que acuden a tratamiento por heroína. De la misma manera, no existen diferencias en la duración del periodo más largo de empleo, pero sí en el periodo más largo de desempleo. Los heroinómanos se pasan más tiempo desempleados que los cocainómanos. Además de esto, la fuente de ingresos más frecuente de los usuarios del programa de cocaína es el empleo, mientras que en el programa de heroína pasa justamente lo contrario, normalmente reciben ingresos a través de familiares, amigos, compañeros, seguridad social, pensiones y fuentes ilegales.

También se encuentran diferencias significativas entre ambos grupos en la mayoría de los ítems que se refieren a la situación legal del paciente. Los heroinómanos han sido acusados de la mayor parte de los delitos que explora el EuropASI en más ocasiones que los cocainómanos y, a su vez, han estado más veces y durante más tiempo en prisión que éstos. Este es un dato relevante puesto que como han encontrado varios estudios, la conducta delictiva previa es una variable que influye directamente en la retención (Gimeno et al., 2002).

En el área de las relaciones familiares son de nuevo los heroinómanos los que refieren mayores problemas. Éstos refieren haber tenido periodos en los que experimentaron problemas serios con familiares, pareja o amigos en mayor medida que los que acuden a tratamiento por sus problemas con la cocaína.

El porcentaje de sujetos que han padecido trastornos psicopatológicos es muy elevado en ambos grupos; pero, a diferencia de otras investigaciones (Craig y Olson, 1990), ninguno de los dos grupos puntúa más que el otro en haber experimentado algún tipo de problema como depresión, ansiedad, alucinaciones, etc., ni en haber acudido a tratamiento por ello. La única diferencia se encuentra en el número de intentos de suicidio. Existe un mayor número de heroinómanos que de cocainómanos que han intentado suicidarse.

En conclusión, los usuarios del programa de heroína presentan un perfil más grave y un mayor deterioro en la mayoría de las áreas evaluadas en el EuropASI: estado de salud, educación y empleo, situación legal y relaciones sociales/familiares. Estos datos coinciden con los obtenidos en estudios similares previos en los que se comparan estos dos tipos de consumidores. Parece confirmarse que el uso continuado de heroína produce un mayor deterioro que el de cocaína en la mayoría de las áreas que se suelen ver afectadas por la dependencia a las drogas. Sin embargo, y a pesar de que la gravedad de la adicción de los heroinómanos es significativamente mayor que la de los cocainómanos, éstos presentan una patología dual igual de grave que los primeros. Este es un dato relevante de cara al manejo clínico de estos pacientes, ya que es un hecho conocido de que las personas con diagnóstico dual presentan una mayor dificultad en el tratamiento y un peor pronóstico.

\section{REFERENCIAS}

Aharonovich, E., Nguyen, H. T. y Nunes, E. V. (2001). Anger and depressive states among treatment-seeking drug abusers: Testing the psychopharmacological specificity hypothesis. American Journal on Addictions, 10(4), 327334.

Ballard, C. A. (2003). Counseling outcome research: The use of the Addiction Severity Index in a homeless population. Univ Microfilms International.

Bobes, J., González, M. P., Sáiz, P. A. y Bousoño, M. (1996). Índice europeo de severidad de la adicción: EuropASI. Versión española. Actas de la IV Reunión Interregional de Psiquiatría, 1996, 201-218.

Brown, T. G., Seraganian, P. y Shields, N. (1999). Subjective appraisal of problem severity and the ASI: secondary data or second opinion? Addiction Severity Index. Journal of Psychoactive Drugs, 31(4), 445-449.

Butler, S. F., Budman, S. H., Goldman, R. J., Newman, F. L., Beckley, K. E., Trottier, D. y Cacciola, J. S. (2001). Initial validation of a computer-administered Addiction Severity Index: the ASI-MV. Psychology of addictive behaviors: journal of the Society of Psychologists in Addictive Behaviors, 15(1), 4-12.

Chermack, S. T., Roll, J., Reilly, M., Davis, L., Kilaru, U. y Grabowski, J. (2000). Comparison of patient selfreports and urinalysis results obtained under naturalistic methadone treatment conditions. Drug and Alcohol Dependence, 59(1), 43-49. 
Claus, R. E., Kindleberger, L. R. y Dugan, M. C. (2002). Predictors of attrition in a longitudinal study of substance abusers. Journal of Psychoactive Drugs, 34(1), 69-74.

Craig, R. J., \& Olson, R. E. (1990). MCMI comparisons of cocaine abusers and heroin addicts. Journal of Clinical Psychology, 46(2), 230-237.

Fieldman, N. P., Woolfolk, R. L. y Allen, L. A. (1995). Dimensions of self-concept: A comparison of heroin and cocaine addicts. American Journal of Drug \& Alcohol Abuse, 21(3), 315-326.

French, M. T., Salome, H. J., Sindelar, J. L. y McLellan, A. T. (2002). Benefit-cost analysis of addiction treatment: methodological guidelines and empirical application using the DATCAP and ASI. Health Services Research, 37(2), 433-455.

Gimeno, C., Esteban, J., Pellin, M. C., Climent, J. M., Camacho, I., Gimeno, M. J., Llinares, R., Furio, J. L., Mora, E., Tores, D., Martin, T. y De La Hoya, P. S. (2002). Mejoria de la gravedad de la adiccion en los tratamientos con metadona. Adicciones, 14(2), 151-160.

Hanlon, T. E., O'Grady, K. E. y Bateman, R. W. (2000). Using the Addiction Severity Index to predict treatment outcome among substance abusing parolees. Journal of Offender Rehabilitation, 31(3), 67-79.

Hubbard, R. L., Flynn, P. M., Craddock, S. G. y Fletcher, B. W. (2001). Relapse after drug abuse treatment. En F. M. Tims y C. G. Leukefeld (Eds.), Relapse and recovery in addictions. (pp. 109-121): Yale University Press.

Johnson, J. L., Wiechelt, S. A., Ahmed, A. U. y Schwartz, R. P. (2003). Outcomes for Substance User Treatment in Women: Results from the Baltimore Drug and Alcohol Treatment Outcomes Study. Substance Use \& Misuse, 38(11), 1807-1829.

Kokkevi, A. y Hartgers, C. (1995). European adaptation of a multidimensional assessment instrument for drug and alcohol dependence. European Addiction Research, 1, 208-210.
Leonhard, C., Mulvey, K., Gastfriend, D. R. y Shwartz, M. (2000). The Addiction Severity Index: a field study of internal consistency and validity. Journal of Substance Abuse Treatment, 18(2), 129-135.

McCaul, M. E., Svikis, D. S. y Moore, R. D. (2001). Predictors of outpatient treatment retention: patient versus substance use characteristics. Drug and Alcohol Dependence, 62(1), 9-17.

McLellan, A. T., Luborsky, L., Woody, G. E. y O'Brien, C. P. (1980). An improved diagnostic evaluation instrument for substance abuse patients. The Addiction Severity Index. The Journal of Nervous and Mental Disease, 168(1), 26-33.

Rosen, C. S., Ouimette, P. C., Sheikh, J. I., Gregg, J. A. y Moos, R. H. (2002). Physical and sexual abuse history and addiction treatment outcomes. Journal of Studies on Alcohol, 63(6), 683-687.

Scheurich, A., Muller, M. J., Wetzel, H., Anghelescu, I., Klawe, C., Ruppe, A., Lorch, B., Himmerich, H., Heidenreich, M., Schmid, G., Hautzinger, M. y Szegedi, A. (2000). Reliability and validity of the German version of the European Addiction Severity Index (EuropASI). Journal of Studies on Alcohol, 61(6), 916-919.

Serrat More, D. (2003). Problemas medico-legales del consumo de drogas. Adicciones, 15(1), 77-89.

Shorin, E. A. (1999). Drug choice, affect., Univ Microfilms International.

Siegal, H. A., Falck, R. S., Wang, J. y Carlson, R. G. (2002). Predictors of drug abuse treatment entry among crackcocaine smokers. Drug and Alcohol Dependence, 68(2), 159-166.

Sindelar, J. L., Jofre-Bonet, M., French, M. T. y McLellan, A. T. (2004). Cost-effectiveness analysis of addiction treatment: paradoxes of multiple outcomes. Drug and Alcohol Dependence, 73(1), 41-50. 
